\title{
EL PLANEAMIENTO URBANO DE MEDIADOS DEL SIGLO XIX: EL PLAN GEOMÉTRICO O DE ALINEACIONES DE ALCOY DE 1849
}

\author{
Juan Manuel Dávila Linares
}

RESUMEN

Los planes geométricos de alineación y rectificación de ciudades, creados por R.O. de 25 de julio de 1846, representaron un claro avance en la "ordenación" de nuestras ciudades, y un evidente aunque mínimo progreso en relación con las prácticas urbanísticas anteriores, basadas en la formulación de cuerpos normativos de ordenanzas de edificación y policía urbana y reglamentos de ornato público. Sin embargo, la ley seguía adoleciendo de una visión mucho más amplia y sobre todo previsiva, centrado su cometido en la apertura de nuevas calles, la rectificación del trazado de aquéllas y la remoción de obstáculos viarios. En este sentido, Alcoy aprobó su Plan de Alineaciones y Rectificación de calles en 1849 , un plan que fue ejecutado muy lentamente y con escasa repercusión en el desarrollo urbano general de la ciudad.

\footnotetext{
ABSTRACT

Geometrical planes of alignment and rectification of cities, created by R.O. of 25th. July of 1846, represented an important advance in the ranking of our cities, and evident although that, minimum progress in relation with the previous urban practicies, based in the formulation of standard body building ordinances and urban police and regulations of public adornment. However, the law continuos to suffer from a vision much wider and above all far-sighted, centred in the commitment in opening new roads, the rectification of the layout of them and removal of obstacles roads. In this way, Alcoy approved their Plan of Alignments and Rectification of roads in 1849, a plan that was carried out very slowly and with hardly any repercussion in the urban general development of the city.
} 


\section{a) Antecedentes}

Si bien durante la segunda mitad del siglo XVIII se llevaron a cabo varios y positivos intentos de dirigir, si no planear, el desarrollo urbano de nuestras ciudades, no será, sin embargo, hasta bien entrado el siglo XIX cuando estos intentos adquieran carta de naturaleza, y ya no sólo a nivel municipal, como hasta entonces, sino provincial e incluso nacional. A las simples reglas de ornato y policía urbana, de ámbito estrictamente local, someras, tendentes tan sólo a regular formas y medidas de fachadas y elementos exteriores de las edificaciones, vendrán a sustituirlas leyes de expropiación forzosa por causa de utilidad pública, ordenanzas de edificación, planes de alineación $\mathrm{y}$, rectificación de ciudades $\mathrm{y}$, sobre todo, desde mediados de siglo, los omnipresentes planes de Ensanche de Poblaciones, y los posteriores de Reforma y Saneamiento Interior.

Esta ausencia de planeamiento general e integrado dará lugar en la mayoría de los casos a un crecimiento desordenado de las ciudades, en especial, a base de agregaciones atomizadas, al ir conformándose pequeñas agrupaciones de casas, más o menos espontáneamente, que, formando en principio barrios extramuros, irán engarzándose al casco urbano paralelamente al progresivo desarrollo espacial de la ciudad central.

La ausencia de un sentido practico de ordenación urbana en Alcoy facilitó así los asentamientos a lo largo de los principales caminos que parten de la ciudad, como el de Alicante -barrio de San Vicente y el de Casas Nuevas-, el de Penáguila- -barrio del Tosal-, el de Concentaina -barrio de Caramanchel- o el de Madrid- barrios del Pla o Santa Elena y de San Sebastián-. Algunos de ellos eran en realidad agrupaciones de naturaleza rural, que fueron quedando integrados en el tejido urbano con el desarrollo de éste durante el siglo XIX, como fue el caso de la -partida de -Caramanchel, que conducía a Concentaina.

Desde comienzos del siglo XVIII. Alcoy conocerá la etapa de mayor crecimiento poblacional de su historia, pasando de 3.800 habitantes a más de 14.600, en tan sólo cien años, este fenómeno provocará de inmediato un creciente interés por edificar en los terrenos todavía no urbanizados que se extendían al sur de la ciudad, al hacerse cada vez más patente el hacinamiento y falta de viviendas en el interior de la misma. Hacia el sur se localizaban los únicos espacios entonces aptos para la edificación, pues las posibilidades de expansión del recinto urbano, tanto por el norte, como por el oeste y el este, se veían interrumpidas por profundos taludes, fruto de los abarrancamientos de los ríos Barchell y Molinar.

Por otro lado, a partir de mediados de siglo, y al igual que en otras ciudades importantes de la provincia, como Alicante y Elche, las autoridades municipales alcoyanas comienzan a plantearse las tareas necesarias para la consecución de un estado urbanístico ordenado y acorde con las crecientes necesidades de viviendas para la población ${ }^{1}$. Sin embargo, estas medidas tan sólo alcanzarán pretensiones menores, como son, simples normas de edificación o modestas actuaciones urbanísticas puntuales.

Al tratarse únicamente de medidas de ámbito local, el control de toda esta ordenación recaía sobre el Ayuntamiento en la persona del Comisario urbano, encargado de recoger, y

1 Cf. DAVILA LINARES, Juan Manuel, "El Urbanismo y la ciudad de Alcoy en el siglo XVIII”, Revista de Fiestas, Alcoy, 1988, pp. 153-155. 


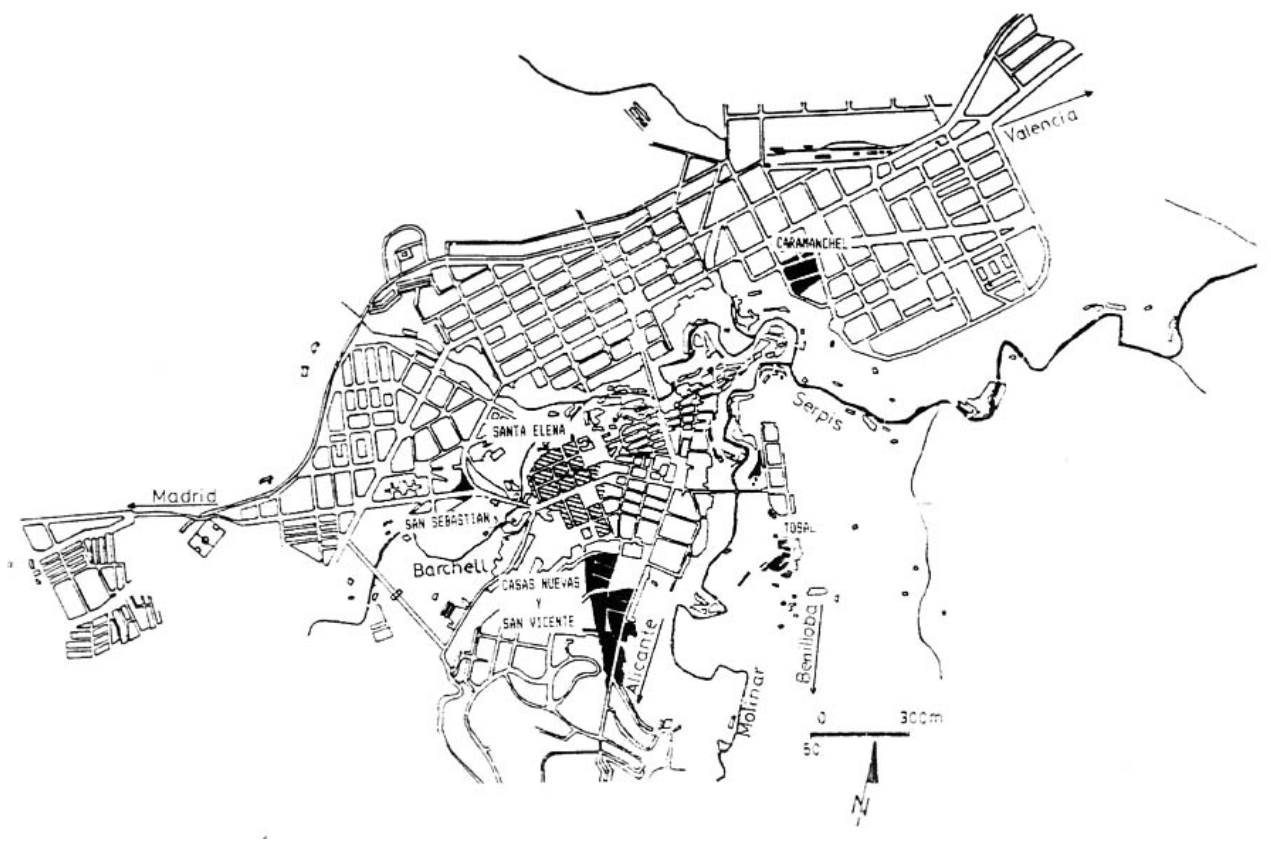

Figura 1: LOCALIZACIÓN DE LOS BARRIOS CAMINEROS A MEDIADOS DEL SIGLO XIX. En negro, situación de los barrios en cada uno de los principales caminos de salida de la ciudad. En rayado, barrio del Pla o Santa Elena, de claro trazado ortogonal y que se convertirá en la primera de la primera de las tres zonas del Proyecto de Ensanche de 1978. 
en su caso, aprobar, los correspondientes permisos de edificación. Estos Comisarios, conocidos también como Policía del Pueblo, estaban facultados para “...derribar, reedificar y quitar lo necesario para la ermosura, y aseo de la Poblacion..."”2.

Las Comisiones o Juntas de Policía Urbana aparecieron en estos primeros años de siglo en la mayoría de las ciudades y poblaciones importantes de todo el país y todas ellas con fines similares: la atención al buen régimen y gobierno de la ciudad, pero carentes de cualquier competencia en ordenación y previsión del desarrollo espacial de aquéllas. De todo lo anterior se desprende la idea que venimos repitiendo hasta aquí: la total falta de visión planificadora y de ordenamiento global de la ciudad, anclada la tarea urbanística en un simple control de alineación y de ornato de fachadas. Esta situación no variará demasiado en las primeras décadas del siglo XIX, ocupado el Ayuntamiento y los responsables de la Comisión de Policía urbana en "...la limpieza, hornato e igualdad de las calles, sin permitir desproporción, para que no se deformase el aspecto público..."”.

El mayor logro de estos primeros años del siglo XIX fue quizá la redacción y posterior aprobación de multitud de ordenanzas y reglamentos, que pretendían formar un cuerpo doctrinal que condujese, en cierta medida, el desarrollo espacial de la ciudad y que controlase aspectos como la tipología edificatoria, servicio y abastecimiento urbanos, limpieza, ornato, e incluso delimitara las competencias entre los distintos cuerpos profesionales dedicados a la construcción: arquitectos, maestros de obras, maestros de albañilería, etc. Las ordenanzas fueron un tiempo el instrumento legal que en manos de los ayuntamientos permitían gobernar el desarrollo y edificación de la ciudad, su policía y ornato ${ }^{4}$.

Todavía dentro de la primera mitad de siglo, el 5 de febrero de 1845, fueron redactadas unas Ordenanzas de Policía Urbana, "para la Leal Ciudad de Alcoy”, aprobadas y ratificadas finalmente por el Gobierno Civil de Alicante en 26 de mayo del mismo año. Este con junto de preceptos era notoriamente más denso y completo que algunos intentos anteriores, aunque sólo en el número de artículos, puesto que el ámbito de temas tratados y la profundidad de su estudio seguía limitado en gran medida a cuestiones de ornato, limpieza y poco más. Estas ordenanzas se dividían en tres títulos:

- título 1: Policía de buen orden (31 artículos).

- título 2: Policía de comodidad y ornato (32 artículos).

- título 3: Policía sanitaria (52 artículos).

Esta sucesiva aprobación de nuevos cuerpos de ordenanzas, si bien no suponía la definitiva "ordenación" del desarrollo de la ciudad y, sobre todo, y lo que es verdaderamente importante, la previsión de la futura expansión de la misma, sí que reflejan el deseo de mejorar estas condiciones urbanísticas y la toma de conciencia de la innegable necesidad de conseguir que el plano de las ciudades, hasta ahora un tanto anárquico y sujeto a multitud de intereses y consideraciones, se desarrolle en función de unas pautas de interés general. De cualquier forma, la adopción de reglamentos generales -ordenanzas y reglamentos de edifi-

2 -AMA, Libros de Cabildos /789-1792, acta de 28-III-1789.

3 -AMA. Licencias de Obras 1754-1870, solicitud de 4-II-1822.

4 -CHUECA GOITIA, Fernando, La destrucción del legado urbanístico español, ed. Espasa-Calpe, Madrid, 1977. p. 55. 
cación-, será la forma más difundida y el modo más específico de constitución urbanística de la ciudad decimonónica ${ }^{5}$.

Con posterioridad a éstos, los sucesivos cuerpos de ordenanzas irán redactándose en función de la legislación vigente en cada momento en materia de urbanismo, esto es, los Planes de Alineación de ciudades y los posteriores de Ensanche de Poblaciones.

\section{b) El Plano Geométrico de Alcoy de 1849}

En la mayor parte de los países europeos, una de las primeras manifestaciones de ordenación y expansión urbanística de las ciudades se lleva a cabo a través de un sistema casi único de alineaciones. Estas aparecen así como la primera técnica urbanística de actuación de conjuntos, bien en relación al recinto urbano preexistente, bien con alguna de sus partes ${ }^{6}$. Según una definición de la época, se le daba el nombre de alineación "a la dirección que se determina han de seguirlas calles, plazas, pasadizos, caminos, etc., y a la cual han de sujetarse todas las construcciones y reedificaciones que se ejecuten en una población", práctica que en Madrid recibía el nombre de "tira de cuerdas"”.

De todo aquel contexto de nuevas normativas urbanísticas surgirá, en 1846, el primer plan general de "ordenación" de ciudades. Un plan que, a diferencia de las simples ordenanzas municipales, se redactará para su aplicación en todo el ámbito nacional. Por Real Orden de 25 de julio de 1846, la Reina ordenó

\footnotetext{
“que los Ayuntamientos de los pueblos de crecido vecindario á juicio de V.S. hagan levantar el plano geométrico de la población, sus arrabales y paseos, trazándolos segun su estado actual en escala de uno por mil doscientos cincuenta; que en el mismo plano se marquen con lineas convencionales las alteraciones que hayan de hacerse para la alineación futura de cada calle, plaza, etc. ....".
}

La R.O. se refería tan sólo a "planos geométricos de poblaciones" y no propiamente a planes municipales de alineaciones, conocidos ya en Francia desde la ley de 16 de septiembre de 1807. En efecto, la finalidad primaria del levantamiento de estos planos era ante todo informativa, con el objeto de evitar posibles conflictos por la construcción de edificios de nueva planta y reedificación de los antiguos, pero no planeaba, en modo alguno, el desarrollo urbanístico, visto posteriormente como conjunto.

Ciertamente, esta ley, si bien representó un cierto avance en la ordenación urbanística de las ciudades, seguía adoleciendo de una visión mucho más amplia y, sobre todo, previsiva. Con ella se logró poco más que la apertura de nuevas calles para mejorar la comunicación con los nuevos barrios o para el servicio de los mismos, la rectificación del trazado de aquéllas y la remoción de obstáculos viarios.

5 -SIGA, Paolo, Historia del Urbanismo. El siglo XIX, 1으. I.E.A.L., Madrid, 1981, p. 54.

6 -BASSOLS COMA, M., Génesis y evolución del derecho urbanístico español: 1812-1956, ed. Montecorvo, s.a., Madrid, 1973, 638 pp., cf. 99-127.

7 -de la CAMARA, M., Tratado práctico de Agrimensura y Arquitectura, Valladolid, 1871, p. 458, cit. por BASSOLS COMA, M., op. cit. id., p. 101.

386.

8 -AMA, Boletín Oficial de la Provincia de Alicante, núm. 352, de 3 de septiembre de 1846, circular núm. 
Estos planes, una vez confeccionados, a escala 1:1.250, debían quedar expuestos al público por espacio de un mes, recogiendo el Ayuntamiento durante este plazo cualquier observación que hubiese sobre el mismo. En el seno del Ayuntamiento, las nuevas alineaciones debían ser fijadas por acuerdo de la mayoría de los concejales y trazadas por un arquitecto titulado. En principio se propuso la terminación de los trabajos en el plazo de un año, finalizado el cual debían ser remitidos al Ministerio de la Gobernación para ser ratificados. No obstante, este plazo, por una u otra razón, rara vez fue cumplido, dilatándose en exceso la elaboración de estos planes.

En algunas ciudades, antes de la publicación de la ley de 1846, se habían comenzado ya los trabajos tendentes al levantamiento de planos geométricos de sus cascos urbanos. Así, por ejemplo, en la ciudad de Alicante, en 1838 se encargó a Manuel Fornés y a Simón Carbonell la realización del plano general de la ciudad y, en 1844, a Rafael Pascual la traza del radio urbano $^{9}$. En Alcoy, motivado por la cada vez más apremiante necesidad de viviendas para la población, en 1841 la Diputación Provincial aconsejó al Ayuntamiento de la ciudad el levantamiento de un Plano General de calles y plazas. Sin embargo, el proyecto no pudo ser llevado a cabo por dificultades económicas del Ayuntamiento.

$\mathrm{Al}$ año siguiente es el propio Gobierno Civil de la provincia quien se interesa por la realización de un Plano General, que comprendiese toda la villa, con una exacta delineación de todas las calles y plazas y sobre el que se fijasen las rectificaciones y alteraciones oportunas, “...lo cual constituiría una garantía para los propietarios de casas y demás fincas urbanas...”. E1 gobierno municipal dio su consentimiento para que se llevase a cabo el proyecto, nombrando a tal efecto una comisión. No obstante, este proyecto tampoco sería realidad sino hasta 1846.

A pesar de no poseer todavía plano general, en los años anteriores a la realización del mismo, el Ayuntamiento no dejó de preocuparse de la alineación y ensanche de sus calles, sobre todo de aquéllas que por su importancia lo requerían, así como de crear otras nuevas. Durante estos años fueron alineadas calles como la Mayor o la de San Antonio, y fueron delineados nuevos espacios urbanos, como el barrio de Santa Elena, en la zona comprendida entre el barranco de la Loba y el puente de Cristina.

El definitivo Plano General de Alcoy comenzó a gestarse a principios de 1846. En ese año, en sesión de 12 de enero, el Ayuntamiento propuso el levantamiento del mismo, encargándolo al arquitecto Jorge Gisbert y Berenguer y autorizado posteriormente por el Gobernador Civil el 15 de julio del mismo año ${ }^{10}$. De esta forma, cuando fue promulgada la ley de 25 de julio, Jorge Gisbert había comenzado ya a elaborar el plano de Alcoy.

El mismo arquitecto, a iniciativa personal, desarrolló este Plano General en varios planes parciales, a mayor escala, cada uno referido a una calle o plaza en concreto y que fueron presentados a la corporación municipal en 7 de septiembre de 1848, para su posterior aprobación por el Ministerio. El conjunto de todos estos planos, recogidos en un solo volumen bajo el título de Plano de la Ciudad de Alcoy en la suma de sus calles por el Académico de Mérito de la Nacional de San Carlos y Arquitecto Mayor honorario del Excmo.

9 -RAMOS HIDALGO, Antonio, Evolución Urbana de Alicante. Tesis Doctoral, 2 t, 1983, cf. f. 569; publicada con el mismo título por el Instituto de Estudios "Juan Gil-Albert”, Alicante, 1984, 323 pp.

10 -CORTES MIRALLES, José, Crecimiento urbano de Alcoy en el siglo XIX, Excmo. Ayuntamiento de Alcoy, 1976, p. 25. 
Ayuntamiento de la ciudad de Valencia, D. Jorge Gisbert y Berenguer. 1849, viene prologado por el propio autor en los siguientes términos:

"Conforme á la R. Orden de 25 de julio de 1846 se dignó V.S. encargarme la formacion del Plano general de la ciudad de Alcoy, comprensivo de sus arrabales y paseos, con el objeto de establecer en el mismo lineas generales fijas y permanentes que marcarán el alineamiento y ensanche de la ciudad, como se proviene en aquella soberana resolucion. Efectivamente, realizados aquellos trabajos, se hallan consignados en el espresado plano y se manifiestan en el mismo con lineas fuertes color carmin, tanto en la parte de ensanche de la ciudad por los tres puntos que le hemos concebido, como en el de la alineación y ensanche de sus calles actuales; todo lo cual va explicado en los terminos que nos han parecido mas claros en el Plano general que se lleva mencionado, al cual nos remitimos con los trabajos en detall que espresa el presente volumen.

»Estos dobles trabajos son indispensables para el objeto é idea que se ha tenido presente al fijar la R. Orden que llevamos mencionada, sin los cuales no podria apreciarse con la debida razon el retiro ó avance de los edificios, porque construido aquel con la escala de mil doscientos cincuenta por uno, no podia el compas tomar los menores retiros de los expresados edificios, bajo cuyo concepto y tratando de darle toda la latitud posible al proyecto que se nos ha encargado, acompañamos el presente volumen, que á darle el nombre que nos ha parecido propio, le titulamos Plano de la ciudad de Alcoy, en la suma de sus Calles, el cual comprende veinte y cuatro láminas sobre las que van colocadas todas las calles conocidas hasta el presente en la ciudad, marcando en cada una de ellas con tinta color carmín las lineas de rectificación, cuyos planos se hayan construidos con sujecion á la escala de Doscientos por uno, y que ademas de apreciarse por la escala referida sus correspondientes latitudes, se hallan estas tambien consignadas en numeros del color arriba expresado, colocados en los extremos y donde mas han parecido conforme en cada uno de ellos, para su completa inteligencia" ${ }^{\prime \prime}$.

De igual forma, este volumen viene acompañado de un listado de las calles, callejones y plazas existentes en aquel momento, que hacen un total de 63.

En 1849 fue aprobado el Plano General de Alcoy, cuatro años antes de que lo fuese el de Alicante. Sin embargo, la efectiva puesta en práctica de este Plan de Alineaciones se llevó a cabo muy lentamente, dadas las muchas dificultades y trabas de todo tipo que los propietarios de los edificios sujetos a rectificación oponían a la ejecución de las obras. Eran muchos los intereses que debían conjugarse a la hora de llevar a cabo estos planes. El primero de ellos era el del sagrado derecho de propiedad, que debía ser garantizado y respetado a la vez que justamente ponderado con la conveniencia pública. Generalmente, estos edificios debían retroceder su fachada a la línea marcada en el plano, lo que suponía en la mayoría de los casos una considerable pérdida de superficie habitable en esos edificios, al retroceder aquélla del orden de 1 a 2 metros. Como quiera que sólo los edificios reedificados totalmente debían ajustarse a la línea marcada en el plano, la picaresca dio lugar a innumerables situaciones donde, para evitar retroceder la fachada, tan solo eran derribados los cuerpos superiores de la edificación, dejando intactos los cimientos y el primer piso,

"Recelosos algunos de los propietarios que suscriben de quedar sin fincas en el momento en que traten de obrarlas se retraen de ello limitándose á repararlas y fortalecerlas por dentro ..."12.

11 -AMA, Plano General.

12 -AMA, Licencias de Obras 1843-1859, informe de 12-VIII-1848. 

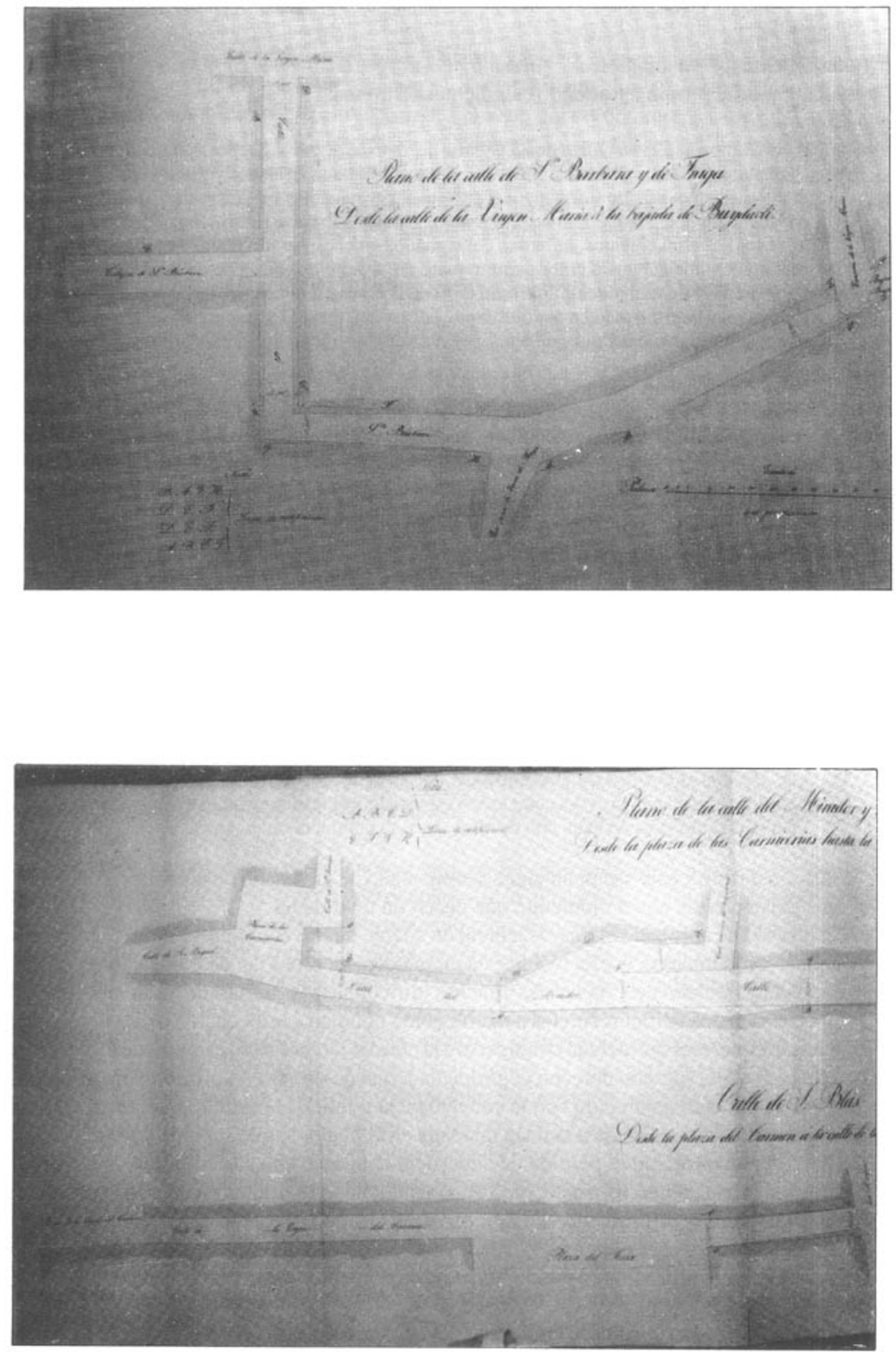

FOTOGRAFÍAS 1 y 2. En los planos de alineación, como los representados en estos fotogramas, el único fin perseguido fue el de marcar sobre el callejero primitivo un nuevo trazado de los viales, más rectilíneo y, en la medida de lo posible, más amplio, pero sin ninguna atención a la superficie edificada, ni a la dotación de servicios, infraestructuras, etc. 
El afán por conjugar, tanto el interés particular, como el del común, se resume en el siguiente informe de la comisión de ornato público, en 3 de noviembre de 1848, en el que se hace referencia al gran número de

“...intereses (que) podrian perjudicarse, en una poblacion de tan numeroso vecindario, y en la que tanto escasea el local para edificar, que al paso que necesita se le proporcione desaogo y ensanche en sus calles no puede perderse de vista la poca capacidad de los edificios particulares, gran parte de ellos lindantes con derrumbadores y terrenos inutiles para edificar de modo que ninguna compensacion pueda tener en cuanto al solar, quedando por lo mismo inutiles á la menor variacion que sufran..."13.

Sin embargo, lo cierto es que las obras de alineación se fueron retardando en demasía. De este hecho son fiel reflejo las licencias de obras para la rectificación y alineación de fachadas, que no aparecen masivamente, sino a partir de 1860-1862. Se justificaba el retardo y la dificultad de llevar a cabo las mejoras expresadas en dicho plan argumentando que la causa principal del retraso era

“...la division de la mayor parte de las casas en varias propiedades, distribuidas por pisos; la dificultad de convenirse los dueños de una misma casa en la epoca de su reedificacion; y la buena calidad de los materiales de que son construidos, y del piso sobre que apoyan, las hace permanecer á pesar de los defectos de construccion de que adolecen y como por otra parte el temor de la retiracion á la linea de ensanche retrae á los dueños aún a los que lo son de toda una casa prefiriendo en su mayor parte hacer las reedificaciones por la parte interior, buscando medios para dar consistencia á sus paredes de fachada sin reedificarlas..."

De hecho, la efectividad de estos planes fue muy escasa, debido sobre todo a que su normativa reguladora se limitaba simplemente a ordenar su formulación, sin preocuparse de prever su contenido material y procedimental ${ }^{15}$.

El primitivo plan de Alcoy fue posteriormente modificado en 1854. Junto al proyecto de modificación del Plano General se acompañaba una relación de aquellas calles cuyo trazado debía ser modificado, contándose un total de 51 calles y dos plazas.

Esta evidente permisividad que la Real Orden de 25 de julio de 1846 tenía con respecto a las obras de alineación y rectificación que venían haciéndose desde su entrada en vigor, había motivado, como se ha visto, primero, el retraso en la culminación de los distintos planes aprobados y, en segundo lugar, una serie de actuaciones que, lejos de favorecer el normal desarrollo de estos planes, lo dificultaban claramente, de forma más o menos sutil, a base de obras de reforma parcial de los edificios, que conseguían que éstos mantuviesen su antigua situación en las calles, sin atenerse a lo prescrito en los Planes de Alineación.

Estos problemas trataron de evitarse con la promulgación primero de la Real Orden de 30 de noviembre de 1857 y más tarde de la de 9 de febrero de 1863, que completaba la anterior. Con ellas se pretendía favorecer la acción del Ayuntamiento en las obras de alineación de sus calles, sin que tuviese que utilizarse cada vez medidas expropiatorias por causa de utilidad pública, lo que a menudo resultaba perjudicial, no sólo para los propietarios, sino también

13 -AMA, Plano General.

14 -AMA, doc. cit. idem.

15 -BASSOLS COMA, M., op. cit., p. 109. 
para los fondos de los municipios. De esta forma, la R.O. de 1863, en su primera disposición, obligaba a todos los propietarios a la línea marcada por el proyecto de alineación de la calle o plaza, según se fuesen demoliendo o reedificando los edificios. Los dueños de las casas sujetas a alineación estaban imposibilitados, asimismo, para efectuar cualquier obra tendente a la consolidación, total o parcial, de las casas, o a cualquier otra que favoreciese la perpetuación de su actual estado, retardando con ello la realización de la mejora proyectada. Preveía también la R.O. la demolición de toda aquella obra que no estuviese construida con estricta y absoluta sujeción al proyecto aprobado y a la licencia concedida, demolición que correría a cargo del propietario (disposición 11). También, serían demolidas las obras de refuerzo o consolidación prohibidas y que venían enumeradas en la R.O. (disposición 12) ${ }^{16}$.

De hecho, la normativa sobre Planes de Alineación influyó bien poco en el crecimiento futuro de las ciudades, limitándose en mayor medida a la rectificación y decoro de lo ya existente. Sin embargo, no cabe duda que iniciativas como ésta y sus respectivas legislaciones supusieron un evidente adelanto en la legislación urbanística del siglo XIX y la paulatina composición de un cuerpo legislativo acorde con las nuevas exigencias y con las disposiciones sobre Ensanches que empezarían a aprobarse a partir de 1864.

16 -AMA, Boletín Oficial de la Provincia de Alicante, núm. 27 de 14 de febrero de 1863. 